Universidad de Lima

Facultad de Psicología

Carrera de Psicología

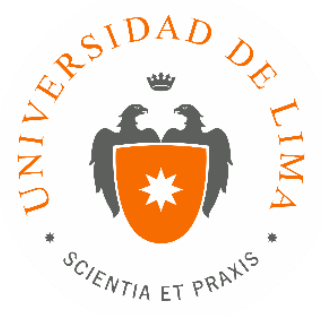

\title{
"EXPERIENCIA DE TRABAJO CON NIÑOS ENTRE LAS EDADES DE 4 A 12 AÑOS EN UN CENTRO TERAPEÚTICO PARTICULAR DE LIMA METROPOLITANA"
}

Trabajo de suficiencia profesional para optar el título profesional de Licenciado en Psicología

\section{Carolina Andrea Jaime Garcia}

Código 20071560

$$
\text { Lima - Perú }
$$

Febrero de 2019 



\section{"EXPERIENCIA DE TRABAJO CON NIÑOS ENTRE LAS EDADES DE 4 A 12 AÑOS EN UN CENTRO TERAPEÚTICO PARTICULAR DE LIMA METROPOLITANA"}




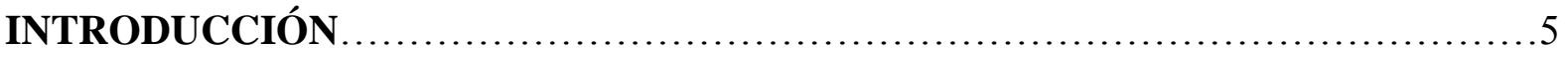

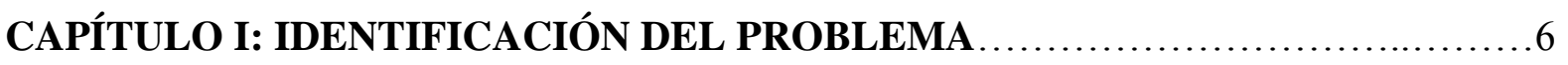

CAPÍTULO II: DESCRIPCIÓN DE LAS ACTIVIDADES REALIZADAS ..............8

2.1 Entrevista clínica con los padres.....................................................

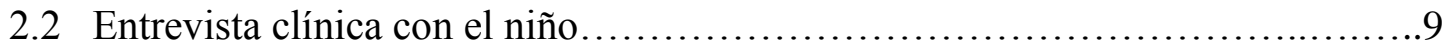

2.3 Devolución del análisis funcional del caso ...................................

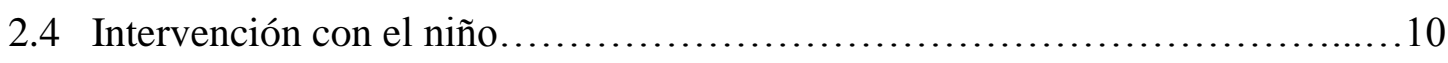

2.4.1 Tratamiento de miedos y temores infantiles............................10

2.4.2 Tratamiento de la impulsividad y desobediencia.........................11

2.4.3 Tratamiento para el Trastorno de Déficit de Atención Con Hiperactividad e Impulsividad (TDAH) ............................11

2.4.4 Tratamiento del Trastorno del Espectro Autista (TEA) con nivel de

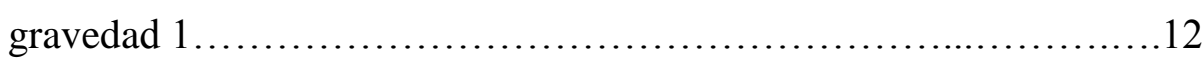

2.5 Entrenamiento en habilidades sociales........................................13

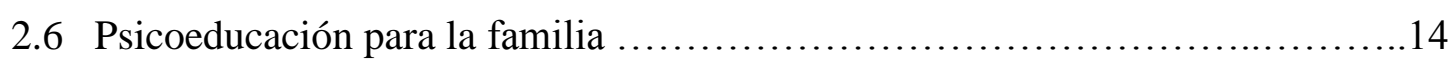

CAPÍTULO III: RESULTADOS DE LA INVERVENCIÓN...............................15

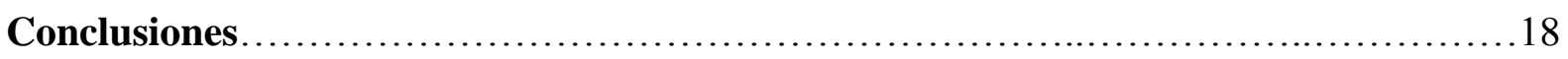

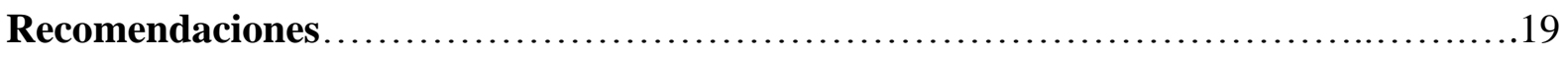

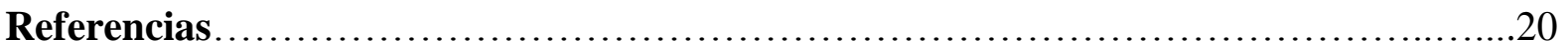




\section{INTRODUCCIÓN}

En el mundo se tiene en cuenta cada día más, la importancia de la detección y prevención de las alteraciones psicológicas infantiles, para facilitar el desarrollo armonioso de la personalidad desde edades muy tempranas (García, 2013). En la actualidad los trastornos emocionales y del comportamiento, forman parte de los grupos de mayor incidencia en las consultas de psicología infantil. Añadido a esto, el Ministerio de Salud (MINSA, 2016) en sus publicaciones, afirma que los trastornos más frecuentes en niños y adolescentes se dan por problemas emocionales, como depresión o ansiedad vinculados a conflictos familiares, seguidos por problemas de conducta, déficit de atención con hiperactividad (TDAH), de aprendizaje y del desarrollo, como el trastorno del espectro autista, entre otros. La consecuencia de éstas, se evidencian en las frecuentes preocupaciones que los padres informan en el motivo de consulta, en las cuales predominan las dificultades para adaptarse a los cambios, problemáticas en la adecuación y rendimiento escolar, incremento de pensamientos de desvalorización personal, tristeza, preocupaciones, miedos, baja tolerancia a la frustración, impulsividad y déficit en habilidades sociales, los cuales pueden repercutir en su adolescencia y adultez si no son atendidos de manera temprana. Ante lo expuesto, es fundamental considerar la importancia y necesidad de atender estas problemáticas que se dan dentro del campo de la salud mental, ya que afectan de manera significativa el desarrollo y funcionamiento general de los niños. La utilidad del presente trabajo de suficiencia profesional permite exponer el desarrollo de las intervenciones terapéuticas abordadas bajo un enfoque cognitivo conductual, validado por la certificación de la Formación Psicoterapéutica en el Instituto Peruano de Terapia Conductual Cognitiva (IPETEC) con la supervisión de psicólogas colegiadas, especializadas en el área infantil. 


\section{CAPÍTULO I: IDENTIFICACIÓN DEL PROBLEMA}

En los últimos años se ha notado un incremento en la asistencia de niños y niñas entre las etapas de primera (0 a 6 años) y segunda infancia (6 a 12 años) a psicoterapia. Van den Heuvel, Barozzino, Milligan, Ford-Jones y Freeman (2016) explican que en la actualidad, los pediatras notan que los problemas de salud mental han reemplazado los malestares físicos, identificando mayor necesidad de atención en el área emocional y comportamental, motivo por el cual los derivan al área de psicología infantil. Esta es una realidad que tiene concordancia con las necesidades de salud mental en el área infantojuvenil de nuestro país. En el Análisis de Situación de Salud Hospitalaria 2015 del Ministerio de Salud (MINSA, 2016), se evidencia que las patologías según CIE 10, que ocasionan mayor atención en la Consulta Externa del Hospital Hermilio Valdizán (HHV), son en primer lugar las Perturbaciones de la Actividad y de la Atención con 1068 casos (22.65 \%), en segundo lugar el Trastorno Hipercinetico, con 779 casos (16.53\%), y finalmente, Autismo en la niñez con 177 (3.75\%).

En el estudio, se concluye que existe una problemática en cuanto a la demanda de atención especializada en salud mental y psiquiatría en el niño y adolescente. Esto conlleva a que los usuarios busquen centros terapéuticos especializados y particulares para poder recibir atención, lo que genera una mayor inversión en comparación con el servicio de salud del estado.

De la misma manera, existen dificultades afines con los pacientes que presentan rasgos del Trastorno de Déficit de Atención con Hiperactividad e Impulsividad - TDAH (DSM V), dado que sus mayores dificultades predominan en la hiperactividad, impulsividad, desatención, baja tolerancia a la frustración y alteración en las funciones ejecutivas, lo que repercute en su autoconcepto, autoestima y autoeficacia, afectando las dimensiones sobre sí mismo, académico, social y familiar. De acuerdo a Rubia, Alegría y Brinson (2014) el TDAH es un desorden del neurodesarrollo, influenciado por múltiples factores genéticos y ambientales. Si 
bien, no existe un dato especifico en nuestro país, el 9.1\% de atención infantil correspondió a los Departamentos de Neuropediatría y Neurología de la Conducta (INCN, 2018).

Por otro lado, en la actualidad existe la demanda de psicólogos con estudios en atención a la diversidad para poder brindar terapia a los niños que presentan el Trastorno del Espectro Autista (TEA) con nivel de gravedad 1 (DSM V). De acuerdo a Arróniz-Pérez y BencomoPérez (2018) el DSM 5, incluye los TEA dentro de la categoría de trastornos del neurodesarrollo, siendo sus principales características la presencia de deficiencias persistentes en la comunicación y en la interacción social, así como la manifestación de patrones restrictivos y repetitivos de comportamiento, intereses o actividad (APA, 2013).

Las problemáticas descritas son los casos clínicos que con mayor recurrencia se atienden en el centro terapéutico particular donde laboro, donde si bien, se atiende a todo el ciclo vital, en el mes de diciembre del año 2018 se atendieron a cuatro niños por cada adulto, apreciándose mayor demanda en el área infantil.

En general es un centro joven, el cual surge de la necesidad de encontrar nuevos espacios de integración para la familia con profesionales especializados en neuropsicología, psicología, aprendizaje, lenguaje y habla, psicomotricidad y ocupacional enfocado en integración sensorial; siendo sus intervenciones tanto individuales como grupales acordes a las necesidades de cada usuario para potenciar y desarrollar sus habilidades, y así contribuir con su crecimiento personal. Cabe mencionar que existe un área social, el cual se basa en atender anualmente a un mínimo de 24 personas de bajos recursos que requieran de alguno de los servicios del centro, por ello cada profesional que ingresa al equipo debe cumplir con la misión, visión y método para que se logren los objetivos institucionales, que a su vez, favorecen el desarrollo de los usuarios. 


\section{CAPÍTULO II: DESCRIPCIÓN DE LAS ACTIVIDADES Y TAREAS REALIZADAS}

El centro donde laboro, ha desarrollado una metodología que incluye la cita de historia clínica, sesiones con el paciente, dos asesorías emocionales y conductuales para la familia y finalmente, un informe con los objetivos y logros alcanzados (Material Propio de la Institución). Esto se realiza en 25 sesiones, dos veces por semana, tomando como modelo la terapia cognitiva conductual. Esta se basa en los aporte de psicólogos como Bandura, Meichenbaum, Kendall, Braswell, Beck y Ellis, tomando como fundamento las leyes de aprendizaje clásico, operante y vicario (Mendoza, 2010). A partir de ello, el Instituto Peruano de Terapia Conductual Cognitiva (IPETEC, 2016, p. 129) la define como:

La TCC utiliza el análisis funcional desde el conductismo paradigmático, en donde por medio de una entrevista clínica permite detectar las relaciones funcionales entre los componente del proceso de aprendizaje, su incidencia en el organismo, la respuesta con sus componentes, motor, fisiológico, emocional y cognitivo, así como las consecuencias de la conducta en contingencias de refuerzo y de castigo, lo que produce la retroalimentación en sus dos formas: la auto retroalimentación y la realizada por otras personas.

Mediante este marco, la que suscribe, brindó atención psicoterapéutica a 22 niños durante el año 2018. Entre ellos, 14 presentaron dificultades emocionales (por ejemplo, reconocimiento de emociones, miedos infantiles y déficit en habilidades sociales), tres presentaron dificultades de impulsividad y desobediencia, dos presentaron TDAH, y tres presentaron TEA. Para ello, se realizaron 83 asesorías emocionales y conductuales a los padres de familia y cuidadores, y alrededor de 50 sesiones de psicoterapia infantil bajo la siguiente estructura. 


\subsection{Entrevista clínica con los padres}

Se inicia con una entrevista de historia clínica con los padres de familia mediante el Cuestionario del Desarrollo (Material Propio de la Institución). Durante ella, se explora el motivo de consulta, se descartan posibles causas orgánicas, y se detecta el origen del problema y las variables relacionadas. Luego, se les entrega la Comunicación a Colegios (Material Propio de la Institución), para ser enviada al colegio y obtener información sobre el desenvolvimiento del niño en su contexto educativo. Si es necesario recabar mayor información, se entregan cuestionarios adicionales a la familia y escuela.

\subsection{Entrevista clínica con el niño}

Se utiliza la entrevista clínica para niños y adolescentes, y observación de conducta junto al S.O.S The TOOL of the BOX (Reategui, 2012). Estas consisten en explorar los cuatro componentes del análisis funcional (cognitivo, fisiológico, emocional y conductual) y estrategias de resolución de conflicto por medio de imágenes y actividades semiestructuradas; este material se complementa con la entrevista clínica infantil (Morales, 2015) (Material Propio de la Institución). Estas herramientas permiten identificar las conductas problemas y necesidades del menor para elaborar el plan de intervención e iniciar con la psicoterapia.

\subsection{Devolución del análisis funcional del caso}

Se les explica a los padres los resultados obtenidos de la entrevista clínica y en función al caso y edad del niño, se le brinda la misma información de manera simple y concreta. Finalizando, se detalla el plan de intervención y los objetivos de trabajo. 


\subsection{Intervención con el niño}

Todo proceso terapéutico comienza con un trabajo de educación emocional, el cual consiste en realizar un entrenamiento inicial en el reconocimiento, identificación y diferenciación de las emociones básicas (alegría, tristeza, enojo y miedo), así como, gestos, posturas y expresiones no verbales relacionadas. Para ello, se utilizan materiales como dibujos, muñecos, títeres, cartillas y memoria de emociones. Posteriormente, se desarrollan ejercicios en base a historias cotidianas y de tipo social para que describan lo que entienden de ellas, de esta manera se les instruye en la comprensión y aceptación de las mismas. También, se les enseña a identificar lo que sienten con ayuda de materiales concretos como termómetros emocionales numéricos, semáforos de caras, etc. (Bunge, Gomar \& Mandil, 2009). Luego, en base al problema específico, se desarrollan intervenciones particulares detalladas a continuación.

\subsubsection{Tratamiento de miedos y temores infantiles}

Si bien es esperable que niños experimenten miedos en diferentes etapas del desarrollo, en algunos casos estos no remiten espontáneamente y perduran en la adolescencia. Estos pueden llegar a interferir con el funcionamiento del niño en el ámbito personal, familiar, escolar, entre otros (Méndez, 2013). En dichos casos, luego del entrenamiento en educación emocional, se desarrolla una jerarquía de miedos para trabajarlos en orden de intensidad. Para cada uno, se realiza un trabajo cognitivo donde se les ilustra las diferencias entre situaciones reales e imaginarias mediante cuentos, secuencia de imágenes y ejemplos de situaciones cotidianas. Cuando logra reconocer la diferencia de ambas, se introducen las técnicas de relajación, respiración e imaginería (Bunge, Gomar, \& Mandil, 2009), para afrontar 
los temores mediante aproximaciones sucesivas. Además, se utiliza el modelado y el reforzamiento positivo para consolidar el aprendizaje de estas herramientas.

\subsubsection{Tratamiento de la impulsividad y desobediencia}

Ciertas conductas, como los berrinches relacionados al comer, dormir, hacer tareas y seguir las indicaciones de los padres son consideradas conductas impulsivas o desobediencia Blackham y Silberman (1971).

Para ellas, se utiliza la modificación de conducta, donde se utiliza el moldeamiento para obtener la conducta deseada por medio del refuerzo positivo o economía de fichas. Además, se utiliza el tiempo fuera o el retiro de un coste de respuesta cuando no cumple con lo establecido. Cuando el niño logra discriminar, aceptar y concientizar los adecuados patrones de conducta, se realiza un entrenamiento en autocontrol, donde se desarrolla en mayor profundidad la emoción de la ira, utilizándose la técnica del Volcán del Enojo y el Termómetro Emocional (Bunge, Gomar, \& Mandil, 2009). Estas herramientas permiten al individuo identificar sus emociones y aplicar técnicas de relajación, respiración, comunicación asertiva y solución de problemas cuando sea necesario, para así regular su conducta de acuerdo a los objetivos establecidos.

\subsubsection{Tratamiento para el Trastorno de Déficit de Atención con Hiperactividad e Impulsividad (TDAH)}

Las personas con inatención tienen dificultades para mantenerse atentas, pierden la secuencia de la actividad, son desorganizados, cometen errores en sus tareas, olvidan y pierden material, y dejan todo para último momento. La hiperactividad es el exceso de actividad verbal o motriz inapropiada para la edad de desarrollo y para la situación 
o tarea; se levantan, merodean sin sentido, trepan, corren o hablan en exceso. La impulsividad es la tendencia a actuar de forma brusca o no planificada, sin reflexión previa, sin considerar las consecuencias para sí mismo o para los demás (Ezpeleta \& Toro, 2014).

Luego del proceso de educación emocional, se realiza un entrenamiento en autocontrol similar al tratamiento de impulsividad y desobediencia, con el objetivo de fortalecer su autoconcepto, autoestima y autoeficacia y puedan realizar una autoevaluación más positiva de ellos mismos. En cada sesión se pone en práctica autoinstrucciones, estrategias de resolución de conflictos, todas estas están disgregadas en Los Programas de Intervención Cognitivo Conductual para Niños con Déficit de Atención con Hiperactividad - TDAH (Orjales \& Polaino-Lorente, 2008) que aún sigue vigente hasta la fecha.

De igual manera, se realiza un entrenamiento en las funciones ejecutivas donde se estimula y potencia las funciones de atención, memoria, y velocidad de procesamiento, de la colección Estimular y Aprender de Jarque (2012), junto a juegos estructurados donde se estimule la captación de información, retención y autocontrol. Asimismo, se hace uso del Programa de Entrenamiento en Planificación (Orjales \& De Miguel, 2017), el cual incluye un sistema de autoinstrucciones para regular la tolerancia a la frustración y al error, seguimiento de indicaciones y atención sostenida.

\subsubsection{Tratamiento del Trastorno del Espectro Autista (TEA) con nivel de gravedad 1}

Los niños con este trastorno requieren de ayuda para mejorar sus deficiencias en la comunicación, apertura e interacción social, dado que pueden tornarse reiterativos y 
literales en su comunicación; además, presentan inflexibilidad de comportamiento, problemas de organización y de planificación que dificultan su autonomía (APA, 2013). Los objetivos básicos en un programa de intervención con personas TEA nivel de gravedad 1 incluyen el logro de habilidades comunicativas (saludar, seguir normas sociales), competencias emocionales (reconocer, comprender, y regular emociones), de interacción (juego funcional y simbólico), de planificación y estructuración de tiempo (planificar tareas y pasos para lograr objetivos), de higiene personal, y de relajación y control conductual (Martínez et al., 2012). Para ello, se hace uso de diversos programas como el Manual de Emociones Básicas (Zardaín, 2013), el Manual de Teoría de la Mente (Cornago, 2012), el Manual de Juego para Niños con Autismo (Cornago, 2012), y pictogramas y tarjetas de secuencia social.

\subsection{Entrenamiento en Habilidades Sociales}

Según Caballo (2010), el entrenamiento en habilidades sociales (EHS) es una de las técnicas más potentes y más frecuentemente utilizada para el tratamiento de problemas psicológicos, siendo fundamental en todo proceso de intervención psicoterapéutica. Ante la necesidad expuesta, Goldstein, Sprafkin, Gershaw y Klein (1989) elaboraron un programa estructurado de habilidades sociales y de autocontrol para niños y adolescentes agrupados en seis apartados de elementos a trabajar. El primero incluye aspectos básicos como presentarse e iniciar una conversación. El segundo incluye saber pedir ayuda, seguir instrucciones, convencer a los demás y disculparse. El tercero se relaciona conocer y expresar los sentimientos propios, y comprender los de los demás. El cuarto tiene que ver con compartir, ayudar, defender los propios derechos, y el autocontrol. Las habilidades para hacer frente al estrés incluyen formular y responder a quejas, responder a la persuasión y al fracaso, prepararse para conversaciones difíciles 
y hacer frente a la presión grupal. Finalmente, las habilidades de planificación suponen tomar iniciativas, establecer objetivos, resolver problemas, tomar decisiones y concentrarse en una tarea. Todas ellas son aplicadas mediante estrategias de moldeamiento, role play, historias para incrementar la comprensión y lectura social, y videos.

\subsection{Psicoeducación para la familia}

Según Morales et al. (2015, p. 28), “para la familia es útil entender qué está ocurriendo, qué conductas favorecen que se sigan manteniendo el problema, tanto por su parte, como por parte del niño. Es necesario que entiendan que culparse o culpar al niño de la situación, o renegar, no son mecanismos adecuados para resolver el problema". Por ello es importante explicarles y entrenarlos en los fundamentos del aprendizaje operante para que logren tener un adecuado manejo de las contingencias y mantener las conductas deseadas en el tiempo.

Por lo tanto se les entrena en ejercicios de autoevaluación, estrategias de modificación de conducta como el refuerzo positivo, economía de fichas, modelamiento y extinción. También se les brinda asesorías emocionales donde se les enseña técnicas de relajación y respiración (Snel, 2013). Asimismo, se les entrena en manejar diálogos positivos y frases de contenido afectivo para brindar un soporte emocional a los niños y puedan así, lograr comunicarse de manera más asertiva. Esto promueve el fortalecimiento de vínculos, lo que ayuda a que los niños puedan tener confianza con los padres para poder expresar sus ideas y pensamientos, miedos y dificultades de tipo social. Todas estas estrategias empoderan y afianzan seguridad en ellos mismos, permitiéndoles tener mayor conciencia de la consecuencia de sus actos, impulsándolos a mejorar por su propio bienestar socioemocional. 


\section{CAPÍTULO III: RESULTADOS DE LA INTERVENCIÓN}

En general, durante el proceso de intervención psicoterapéutico se dio de alta a nueve niños de los cuales se observaron cambios en sus conductas, verbalizaciones, así como, la creación de pensamientos alternativos que los empoderó hacer frente sus temores, impulsividad y dificultades socioemocionales. El entrenamiento continuo permitió que las familias y escuela notasen la transferencia del aprendizaje en sus diferentes entornos.

Actualmente, siguen dentro del proceso terapéutico 13 niños, de los cuales seis de ellos asisten en una frecuencia de una vez por semana debido a los avances alcanzados; cuatro de ellos aún se encuentran en proceso y los otros tres han iniciado psicoterapia en el mes de diciembre del año 2018, por lo que también asisten en una frecuencia de dos veces por semana.

En cuanto a los pacientes que asisten una vez por semana, demuestran haber alcanzado los objetivos de trabajo, encontrándose en una etapa de entrenamiento y exposición para transferir su aprendizaje a diferentes contextos. Uno de los casos, inicio terapia sintiendo temor, angustia y preocupación cuando se tardaban en recogerlo del colegio, pensaba "me van a robar", "se olvidaran de mí y me dejaran en el colegio", "habrá un temblor y no podrán recogerme”, "mi mami, papi, mami lu y papa lucho van a chocarse y no podrán recogerme” etc., experimentando sensaciones fisiológicas como dolor de cabeza y dificultad para respirar; llegando a quedarse inmóvil y en ocasiones, lloraba de manera descontrolada. Ha aprendido a tener control de su cuerpo, llegando a utilizar de manera autónoma las técnicas de relajación y respiración, redirigiendo su pensamiento con auto-instrucciones de empoderamiento. Hasta la fecha, ha demostrado controlar su impaciencia y nerviosismo, esperando con calma que lo recojan del colegio; si bien pregunta quien lo recogerá, verbaliza que ya sabe darse cuenta qué pensamientos le hacen daño y los aleja hablándoles de manera firme porque es un niño fuerte y valiente. Todavía necesita repasar los ejercicios enseñados para consolidar su aprendizaje. 
En cuanto a la intervención de niños con TDAH, se observan mejoras en el manejo de su autocontrol. Uno de ellos, presentó resistencia para utilizar el borrador cuando se equivocaba en sus dibujos o tareas escolares, cuando fallaba reaccionaba de manera impulsiva rompiendo las hojas, gritando y llorando, en esos momentos verbalizaba “¡me tiene que salir perfecto!”, "soy malo en todo" y "ya no dibujare nunca más", quedándose sentado mirando el papel o cruzaba los brazos y agachaba la cabeza en la mesa negándose a culminarlo. Asimismo, le era difícil mantener continuidad en sus trabajos, permaneciendo en ellas aproximadamente cuatro minutos. Dentro del proceso terapéutico ha aprendido a tener un mejor manejo de su autocontrol, logrando calmarse con menos apoyo del adulto. Reconoce que no todo puede ser perfecto, valorando más su esfuerzo, presentando un poco más de tolerancia cuando falla en sus ejecuciones. Es capaz de pedir ayuda con un tono de voz tenue respetando las normas sociales y busca mejorar su trabajo en la misma hoja aceptando su error buscando soluciones adaptativas. Por sus avances, ha logrado focalizar mejor su atención, llegando a iniciar, mantener y culminar los ejercicios psicoterapéuticos y escolares, donde se evidencia mejor capacidad de retención y agilidad visomotora. Si bien, la familia se muestra comprometida con la intervención, en algunas ocasiones no han respetado los acuerdos terapéuticos, siendo necesario monitorearlos semanalmente para evitar retrocesos en los avances del menor.

Por otro lado, los avances de los TEA nivel de gravedad 1 son paulatinos y progresivos, observándose mejoras en la flexibilidad al cambio, contacto visual, comprensión de las emociones y el de las demás, y control conductual. Han aprendido hacer un mejor uso de las habilidades sociales primarias para poder entablar una conversación y comunicar sus ideas, así como aceptar la opinión de los demás. Por su pensamiento literal, son estrictos al momento de usar las técnicas de relajación por lo que se realizan con material concreto para que logren dominar sus impulsos. Requiere de un entrenamiento riguroso en lectura y comprensión social 
por lo que su tiempo de intervención puede ser un poco más duradero, esto dependerá de los recursos del paciente y la colaboración de la familia.

En general, los avances psicoterapéuticos de los pacientes actuales, se encuentran evolucionando favorablemente. Si bien la evaluación cualitativa del progreso la realiza el profesional a cargo del caso, se corrobora la transferencia del aprendizaje con el neuropediatra, docentes y psicólogos educativos. Por ello, la participación de la familia y cuidadores es fundamental ya que son agentes de cambio para el beneficio de los menores.

Es importante hacer mención que, de acuerdo a las necesidades del caso, luego de realizar un periodo de psicoterapia individual, se recomienda asistir a talleres grupales de habilidades sociales o conductuales para que pongan en práctica lo aprendido y puedan desempeñarse con seguridad, para luego aplicarlos de manera independiente y sin apoyo terapéutico.

Finalmente, ante lo expuesto, se continúa corroborando la efectividad de la metodología de la TCC en el área de psicología clínica infantojuvenil, ya que se pueden observar y apreciar avances significativos en los diferentes trastornos del neurodesarrollo y de problemáticas de tipo emocional - conductual. Sus bases de aprendizaje operante y vicario, permiten que el proceso de psicoeducación a los familiares y cuidadores, sea más efectivo por su fácil comprensión y temporalidad. 


\section{CONCLUSIONES}

- La propuesta metodológica de 25 sesiones, de una asistencia de dos veces por semana, que ofrece el centro, evidencia avances significativos en niños entre los cuatro y doce años con trastornos del neurodesarrollo y problemas de tipo emocional-conductual.

- La TCC brinda estrategias efectivas con base empírica, en el abordaje terapéutico de niños con trastornos del neurodesarrollo.

- Poseer estudios de especialización en formación psicoterapeuta cognitiva conductual permite realizar un trabajo profesional, estructurado y responsable, brindándole un servicio de calidad al usuario, basado en evidencia empírica.

- La psicoeducación es un proceso fundamental para obtener resultados en mediano o corto plazo con la población infantojuvenil, ya que sin el apoyo de la familia y escuela, es más trabajoso y extenso el tiempo de psicoterapia con el menor.

- El material del S.O.S the TOOL of the Box (Reategui, 2012) que incluye un modelo de entrevista clínica para niños y adolescentes, es una herramienta útil, creativa e innovadora para explorar de manera dinámica y llamativa los pensamientos, sentimientos, conductas y estrategias compensatorias del paciente.

- En relación a los pacientes atendidos, la totalidad de ellos ha evidenciado mejoras a nivel individual y social, dentro del entorno escolar y familiar, corroborado por los padres y cuidadores, docentes y psicólogos educativos. Es así que nueve de ellos han sido dados de alta y 13 continúan evidenciando avances observables en su proceso terapéutico. 


\section{RECOMENDACIONES}

A continuación se detallarán las recomendaciones:

- Continuar utilizando la metodología dentro del centro terapéutico, debido a que se obtienen avances favorables en mediano plazo.

- Los psicólogos clínicos deben tener estudios en formación psicoterapéutica para poder afrontar las diferentes casuísticas y abordarlas con objetividad, ética y profesionalismo.

- Los psicólogos deben de tener estudios en atención a la diversidad para poder brindar asesorías emocionales y conductuales basado en evidencia empírica, a los familiares y cuidadores de niños con TDAH y TEA.

- Dado a su utilidad y efectividad en los casos atendidos, se recomienda que se implemente el material de S.O.S the TOOL of the BOX (Reategui, 2012) junto a la entrevista clínica para niños y adolescentes en las intervenciones de psicología infantojuvenil del centro, dado que permite recolectar información de manera dinámica y atractiva para el niño. 


\section{REFERENCIAS}

American Psychiatric Association (APA; 2013). Diagnostic and statistical manual of mental Disorders $(D S M-V)$. Washington, D.C.: APA.

Arróniz-Pérez, M. \& Bencomo-Pérez, R. (2018). Alternativas de tratamiento en los trastornos del espectro autista: una revisión bibliográfica entre 2000 y 2016. Revista de Psicología Clínica con Niños y Adolescentes, 5(1), 23-31. DOI: 10.21134/rpcna.2018.05.1.3

Blackham, G., \& Silberman, A. (1971). Modification of Child Behavior. Belmont: Wadsworth.

Bunge, E., Gomar, M. \& Mandil, J. (2009). Terapia Cognitiva con Niños y Adolescentes. Aportes técnicos. Buenos Aires: Librería Akadia Editorial

Caballo, V. (2010). Manual de evaluación y entrenamiento de las habilidades sociales (9na ed.). Madrid: Siglo XXI de España Editores

Cornago, A., Navarro, M. \& Collado, F. (2012). Manual del juego para niños con autismo. Valencia: PSYLICOM Distribuciones Editoriales.

Cornago, A., Navarro, M. \& Collado, F. (2012). Manual de teoría de la mente para niños con autismo. Valencia: PSYLICOM Distribuciones Editoriales.

Ezpeleta, L., \& Toro, J. (2014) Psicopatología del desarrollo. Madrid: Ediciones Piramide

García A. (2013). Psicología clínica infantil. Su evaluación y diagnóstico. La Habana: PSICOalternativas.

Goldstein, A., Sprafkin, R., Gershaw, N., Klein, P. (1989). Habilidades sociales y autocontrol en la adolescencia. Un programa de enseñanza. Barcelona: Ediciones Martínez Roca.

Instituto Nacional de Ciencias Neurológicas (INCN; 2018). Morbimortalidad e indicadores de gestión. Lima: Instituto Nacional de Ciencias Neurológicas. Recuperado de: http://www.incn.gob.pe/images/ESTADISTICAS/10_MORBIMORTALIDAD_2015. pdf

Jarque, J. (2012a). Estimular la Atención: Niveles 4, 5, 6, 7, 8, 9 y 10. Madrid: Gesfomedia. Jarque, J. (2012b). Estimular la Memoria: Niveles 2, 3, y 4. Madrid: Gesfomedia.

Jarque, J. (2012c). Estimular la Velocidad de Procesamiento: Niveles 2, 3, y 4. Madrid: Gesfomedia.

Jongh De. (2017). Psicoterapia infantil: elementos distintivos y propuestas de intervención. 
Revista Alternativas en Psicología, 37. Recuperado de: http://www.alternativas.me/attachments/article/142/Alternativas\%20en\%20Psicolog\% C3\%ADa\%2037\%20-\%20Febrero\%20-\%20Julio\%202017.pdf

Méndez, F. (2013). Miedos y temores en la infancia. Ayudar a los niños a superarlos. Madrid: Ediciones Piramide.

Mendoza, B. (2010). Manual de autocontrol del enojo. Tratamiento cognitivo - conductual. México: El Manual Moderno.

Ministerio de Salud (MINSA; 2016). Análisis Situacional de Salud Hospitalaria. MINSA: Lima.

Ministerio de Salud (MINSA; 2018). Análisis Situacional de Salud Hospitalaria. MINSA: Lima.

Morales, C., García, M., Álvarez, C., Gervás, M., Pardo, R., Pérez, O., \& Santacreu, M. (2015). Guía de Intervención Clínica Infantil. Universidad Autónoma de Madrid.

Orjales, I. \& De Miguel, M. (2017). Programa de Entrenamiento en Planificación. Valencia: CEPE.

Orjales, I. \& Polaino-Lorente, A. (2008). Programa de intervención cognitivo conductual para niños con déficit de atención con hiperactividad (TDAH). Madrid: CEPE

Piazza, M., \& Fiestas, F. (2014). Prevalencia anual de trastornos y uso de servicios de salud mental en el Perú: resultados del estudio mundial de salud mental, 2005. Revista Peruana de Medicina Experimental y Salud Publica, 31(1), 30-8. DOI: 10.17843/rpmesp.2014.311.5.

Reategui, M. (2012). S.O.S. The TOOL in the Box. Lima: INFATEC.

Rubia, K., Alegría, A., \& Brinson, H. (2014). Anomalías cerebrales en el trastorno por déficit de atención/hiperactividad: una revisión. Revista de Neurología, 58(S1), S3-S18.

Snel, E. (2013). Tranquilos y Atentos Como Una Rana. Barcelona: Editorial Kairós.

Van den Heuvel, M., Barozzino, T., Milligan, K., Ford-Jones, E., \& Freeman, S. (2016). We need psychologists! Paediatrics \& Child Health, 21(1), 1-3. DOI: 10.1093/pch/21.1.e1

Velarde, M. Vattuone, J., Gomez, M., \& Vilchez, L. (2017). Nivel de conocimiento sobre Trastorno por Déficit de Atención con Hiperactividad enmédicos del Servicio Rural y Urbano Marginal de Salud en Lima, Perú. Revista de Neuropsiquiatría 80(1), 3-11.

Zardaín, P. (2013). Leo TEAyuda. Las emociones básicas. Manual para la evaluación y enseñanza de emociones. Valencia: PSYLICOM Distribuciones Editoriales. 